\title{
An improved non-local means filter for color image denoising
}

Gaihua Wang, Yang Liu, Wei Xiong and Yan Li

The self-archived postprint version of this journal article is available at Linköping University Institutional Repository (DiVA):

http:/ / urn.kb.se/ resolve?urn=urn:nbn:se:liu:diva- 152407

N.B.: When citing this work, cite the original publication.

Wang, G., Liu, Y., Xiong, W., Li, Y., (2018), An improved non-local means filter for color image denoising, Optik (Stuttgart), 173, 157-173. https:// doi.org/ 10.1016/j.ijleo.2018.08.013

Original publication available at:

https:/ / doi.org/ 10.1016/j.ijleo.2018.08.013

Copyright: Elsevier

http:// www.elsevier.com/

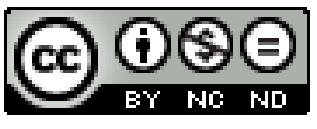




\section{Accepted Manuscript}

Title: An improved non-local means filter for color image denoising

Authors: Gaihua Wang, Yang Liu, Wei Xiong, Yan Li

PII: $\quad$ S0030-4026(18)31126-4

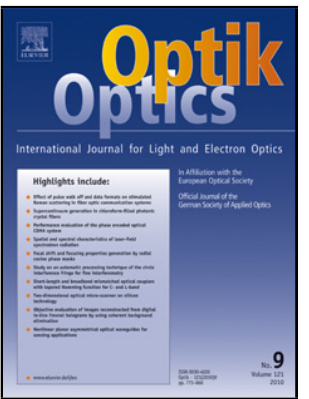

DOI: $\quad$ https://doi.org/10.1016/j.ijleo.2018.08.013

Reference: $\quad$ IJLEO 61310

To appear in:

Received date: $\quad 21-2-2017$

Revised date: $\quad$ 7-8-2018

Accepted date: $\quad$ 8-8-2018

Please cite this article as: Wang G, Liu Y, Xiong W, Li Y, An improved non-local means filter for color image denoising, Optik (2018), https://doi.org/10.1016/j.ijleo.2018.08.013

This is a PDF file of an unedited manuscript that has been accepted for publication. As a service to our customers we are providing this early version of the manuscript. The manuscript will undergo copyediting, typesetting, and review of the resulting proof before it is published in its final form. Please note that during the production process errors may be discovered which could affect the content, and all legal disclaimers that apply to the journal pertain. 


\title{
An improved non-local means filter for color image denoising
}

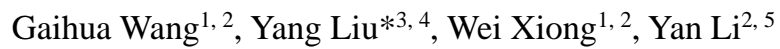

*Corresponding author: Tel: +46 73661 7727; Email: yang.liu@ liu.se

${ }^{1}$ Hubei Collaborative Innovation Center for High-efficiency Utilization of Solar Energy, Hubei University of Technology, Wuhan 430068, China

${ }^{2}$ School of Electrical and Electronic Engineering, Hubei University of Technology, Wuhan 430068, China

${ }^{3}$ Department of Management and Engineering, Linköping University, SE-581 83 Linköping, Sweden ${ }^{4}$ Faculty of Technology, University of Vaasa, PL 700, 65101 Vaasa, Finland

${ }^{5}$ Faculty of Health, Engineering and Sciences, University of Southern Queensland, QLD 4350, Australia

\begin{abstract}
Non-local means filter is a special case of non-linear filter. It performs well for filtering Gaussian noise while preserving edges and details of the original images. In this paper, we propose an improved filter for color image denoising based on combining the advantages of non-local means filter and bilateral filter. To compare the similarity of patches, a new weight value is computed by adding texture information into weights. The experimental results of color image filtering show that the proposed method has a better performance for reducing Gaussian noise and mixture noise.
\end{abstract}

Key words: non-local means; bilateral filter; Gaussian noise; color image denoising 


\section{Introduction}

Color images are widely used but are often corrupted by noises that come from the image acquisition process, transmission or storage. The types of image noise are mainly Gaussian noise, impulse noise and mixed noise. For impulse noise, vector median filter (VFM) [1], vector directional filter (VDF) [2], and directional distance filter (DDF) [3] are well-known classical non-linear filters that have been widely used. However, these filters are spatially invariant operators that make no distinctions between noisy and noise-free pixels. To avoid damaging clean pixels in color images, adaptive vector median filter (AVMF) [4,5], fast peer group filter (FPGF) [6,7] and vector lower-upper-middle smoother (VLUM) [8] are widely used by certain switching schemes. Moreover, These filters are computationally efficient and perform reasonably well in removing noise. Following the success of switching filtering schemes in general imaging, several studies have been conducted to remove the random-valued impulse noise [9-12], but all these median methods aim to remove the impulse noise. When confronting with Gaussian noise, the performances of those methods would be dramatically dropped.

Unlike the local means filter, a non-local means (NLM) filter operates on a non-local area by using a dissimilarity measure between patches. It works well for removing Gaussian noise [13]. Many algorithms based on the NLM technique have been proposed to trade off detail preservation against noise reduction. $\mathrm{Li}$ and Suen [14] analyzed the structure similarity by grey relation of coefficients, and set similar weight function accordingly. Zheng el al. [15] proposed a non-local selection scheme of regularization parameters, which is based on a content-aware function through discriminating image contents. Chen el al. [16] proposed incorporating circulant similarity into a weighted average filter and choosing an appropriate kernel as weight function. To avoid the damage of edge pixels, Bhujle and Chaudhuri [17] proposed matching of both intensity and edge patches, and edge maps were extracted for the neighborhoods. Torres et al. [18] selected homogeneous pixels in the filtering area through statistical tests between distributions, and the weights of the location-variant linear filter are function of p-values of the tests. Sun et al. [19] established a general non-local denoising model based on multi-kernel-induced measures (GNLMKIM). The solutions based on NLM can remove Gaussian noise effectively, but the computational time is longer, and the cost is larger. Meanwhile, blurring still exists and blocking artifacts are also produced. To solve these problems, Wang et al. [20] uses fuzzy membership value to describe the property of pixels and pixels are defined between noises and noise-free, and combined the advantages of fuzzy decision filter (FDF) and NLM filter for noise suppression and edge preservation (FDNLM). Liu et al. [21] propose a NLM filter based on quaternion representation of color images. In addition, the papers $[22,23]$ efficiently remove noise by using a faster NLM filter and show superior denoising performance for clinical images. In addition, to solve over-smoothing of the image or inadequate noise removal, Verma et al. [24] select an optimal size of search window that vary from region to region based on the characteristics of the search region. In addition, the methods $[25,26]$ based on sparse and redundant representations also applied to NLM. And the computationally scalable algorithms can achieve better denoising performance 
for Gaussian noise.

The bilateral filter (BILF) [27] is another non-linear filter that smooths images while preserves edges. Its application can be found in many image processing systems. Pinto et al. [28] introduced a robust bilateral and temporal filter (RBLT) using the spatial and temporal evolution of sequences to guide the filtering process. Shao et al. [29] presented an adaptive fast bilateral filter (AFBF) to reduce the speckle noise. In [30], through the filtering operation of the joint three-dimensional histogram, the target image is processed by jointing bilateral band filter. In [31], a rough set theory based approach is used to derive pixel level edge map and class labels which in turn are used to improve the performance of BILF. The main principle based on BILF extends the concept of Gaussian filter and combines spatial and intensity similarities.

Above all, the weights of the related NLM are all calculated by only using pixel information to measure the similarity. The results of noise removal are not accurate. There are also some methods $[32,33,34]$ that use contourlet Domain rather than in pixel domain. But most algorithms are only for certain noise, impulse noise or Gaussian noise. For the BILF, the weights can use both the spatial similarity and the pixel similarity. In this paper, a new method is proposed. We take the advantages of NLM and BILF, and add a weight value that describes the property of the interested area. The proposed filter can preserve the fine details and edges more effectively. And we also give more experimental result in different noise. The proposed method can remove impulse noise and Gaussian noise effectively.

The rest of this paper is structured as follows. In Section 2, we briefly review the related research work. In Section 3 we describe the methodology of improving the NLM in details. In Sections 4, we present the experiments and results. Finally, we conclude the paper in Section 5 .

\section{Related work}

\subsection{The NLM}

The NLM method was proposed by Buades et al. [13]. Under the Gaussian noise assumption, the weights are calculated, which are used to measure the similarity between a central region patch and neighboring patches in a searching window. A color RGB image $f$ is commonly represented as an array of $H \times W \times 3$, where every pixel is a three-component vector of integer values in the interval of $[0,255]$ (that is $k=3$, representing three channels).

$$
\begin{aligned}
& g_{k}(x, y)=\frac{\sum_{u, v \in N} f_{k}(x+u, y+v) w(u, v)}{\sum_{u, v \in N} w(u, v)} \\
& w(u, v)=\exp \left(-\frac{\sum_{p \in P}\left\|f_{k}(x+p, y+p)-f_{k}\left(x_{0}+p, y_{0}+p\right)\right\|_{2}^{2}}{h^{2}}\right)
\end{aligned}
$$

The NLM method is represented as Equations (1) and (2). $N$ is the searching window. $P$ is the patch window. $\mathrm{h}$ is the parameter to control the degree of smoothness. $g_{k}$ is the filtering result. 
$w(u, v)$ is the weight that uses pixel information to measure the similarity between a central region patch and its neighboring patches (Equation (2)). $\|\bullet\|_{2}$ is Euclidean distance. $\left(x_{0}, y_{0}\right)$ is the position of central pixel, $(x, y)$ is the position of neighboring pixel in the searching window. Each pixel is filtered by Equation (1).

Fig.1 shows different filter results. Fig.1 (a) is the original image from Matlab. Fig.1 (b) is polluted with Gaussian noise with mean 0 and variance 0.01. Fig.1(c)-(f) are filter results with different searching window. Although increasing the searching size $N$ can marginally enhance the denoising performance, it also incurs unwanted computational costs, and blocking artifact is increasing. The selection of the searching size $N$ can be empirical, depending on specific applications. Fig.2 (a) shows the computational time. The computational time increases drastically while increasing the searching window size.

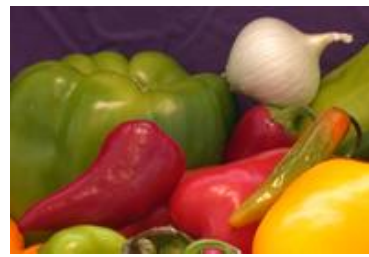

(a)

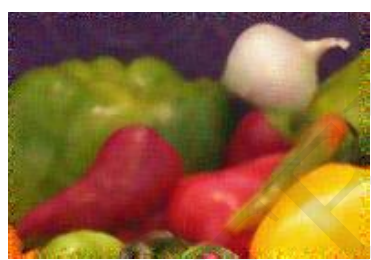

(c)

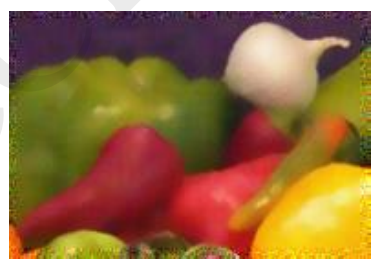

(b)
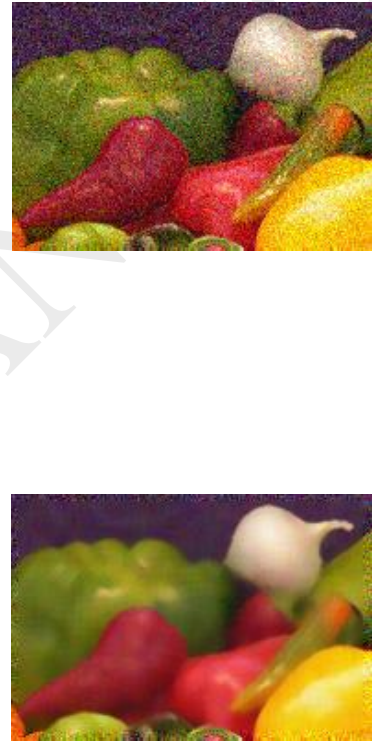

(d)

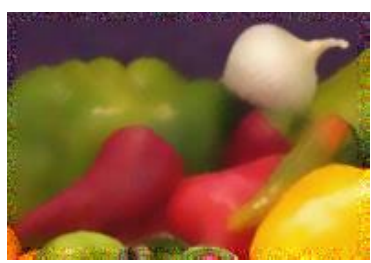

(e)

(f)

Fig.1 the performance of with different searching window: (a) the "onion" original image (b) the "onion" image with Gaussian noise (c) the filter result with a $5 \times 5$ searching window (d) the filter result with a $7 \times 7$ searching window (e) the filter result with a $9 \times 9$ searching 
window (f) the filter result with a $11 \times 11$ searching window

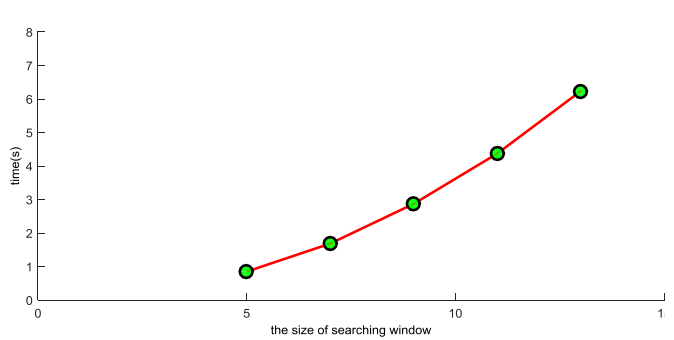

(a)

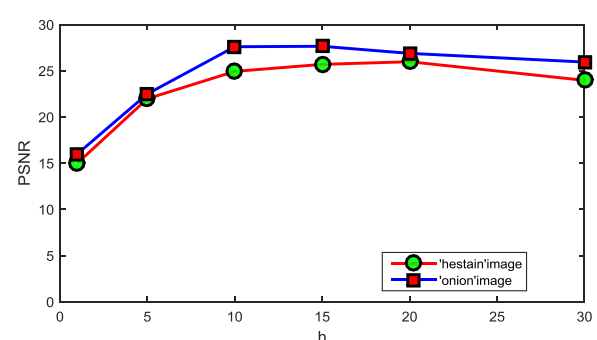

(b)

Fig.2 the performance of the filtering result: (a) the computational time with "onion" image. (b) the filtering performance with different $h$

$\mathrm{h}$ is the parameter to control the degree of smoothness, and several parameters of the NLM filter can be adjusted to achieve the best deblocking effects. We use the NLM filter with different parameter value of $h$ at 5, 10, 15, 20, 30. Fig.2 (b) shows the filtering performance with different $\mathrm{h}$. The original image is corrupted with mixture noise that combines $3 \%$ impulse noise and Gaussian noise of variance 0.01. It is shown that the peak signal-to-noise ratio (PSNR) is lower, and blocking artifacts cannot be efficiently suppressed when $h=5$. The detailed information would be largely lost if it is deblocked by a smoothing filter with $\mathrm{h}>30$. Even if a moderate $h$ is adopted, for example $h=15$, deblocking still cannot bring acceptable results on the whole image. The NLM cannot remain at high effectiveness in removing Gaussian noises as shown in the experiment results. While the NLM fails to remove the common mixed noise, therefore, the denoising performance of the NLM still needs to be improved.

\subsection{The BILF}

The original function of the BILF is to preserve edges while smoothing out images. It is a non-linear filter that has been improved from Gaussian filter. The denoising image is also computed as a weighted average of each pixel's neighborhood [35].

$$
\begin{gathered}
g_{k}\left(x_{0}, y_{0}\right)=\frac{1}{\sum_{(x, y) \in \Omega\left(x_{0}, y_{0}\right)} W\left(x, y, x_{0}, y_{0}\right)} \sum_{(x, y) \in \Omega\left(x_{0}, y_{0}\right)} W\left(x, y, x_{0}, y_{0}\right) f_{k}(x, y) \\
\Omega\left(x_{0}, y_{0}\right)=\left\{(x, y)|| x-x_{0} \mid \leq w_{0} \text { and }\left|y-y_{0}\right| \leq w_{0}\right\} \\
w\left(x, y, x_{0}, y_{0}\right)=\exp -\left(\frac{\left\|f_{k}(x, y)-f_{k}\left(x_{0}, y_{0}\right)\right\|_{2}^{2}}{2 h_{1}^{2}}\right) \operatorname{ex~}-\left(\frac{\left(x-x_{0}\right)^{2}+\left(y-y_{0}\right)^{2}}{2 h_{2}^{2}}\right)
\end{gathered}
$$

$g_{k}\left(x_{0}, y_{0}\right)$ is the filtering result (Equation (3)) which filters images through the combination

of spatial and range Gaussian kernels (Equation (5)). $W_{0}$ (Equation (4)) is the distance between central pixel and neighboring pixel. $f_{k}\left(x_{0}, y_{0}\right)$ is the central pixel, and $f_{k}(x, y)$ is a neighboring pixel. $w\left(x, y, x_{0}, y_{0}\right)$, defined in Equation (5) is the weight value. $w\left(x, y, x_{0}, y_{0}\right)$ is combined the weight based on the similarity between pixel $(x, y)$ and pixel 
$\left(x_{0}, y_{0}\right)$ with the weight based on Euclidean distance between those two pixels. Thus, the

BILF has two free parameters, $h_{1}$ and $h_{2}$. These parameters make it possible to control the influence of the geometric distance and similarity properties of the neighbors, respectively. Fig. 3 shows the influence of the geometric distance and similarity properties of the neighbors with 'onion' and 'hestain' image. The original image is influenced with mixture noise that combines 3\% impulse noise and Gaussian noise of variance 0.01 .

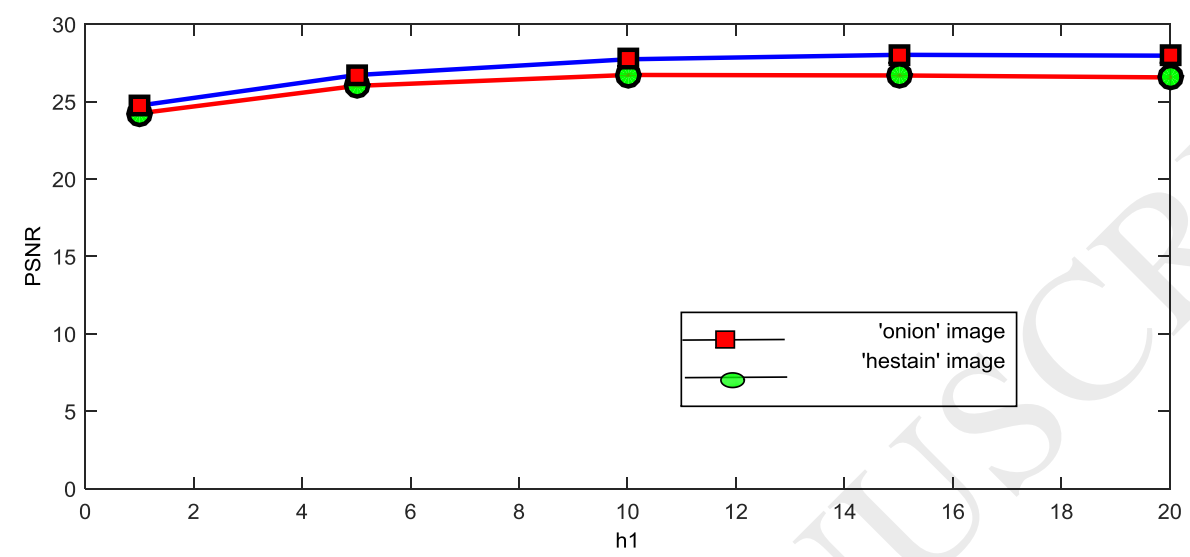

(a)

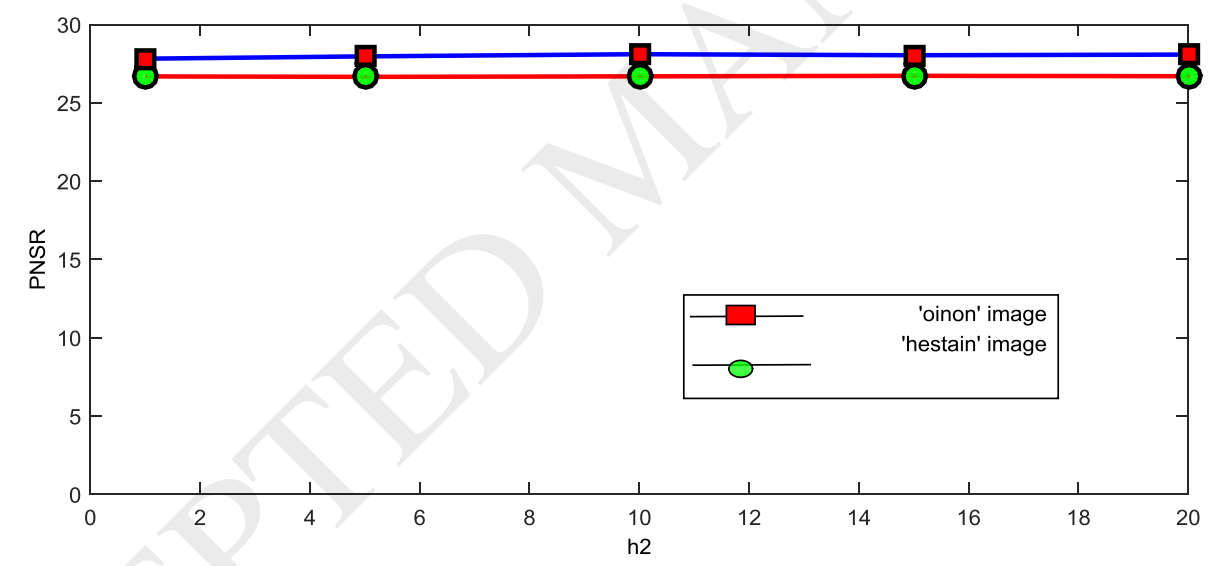

(b)

Fig.3 the filtering performance with different parameters: (a) the performance with various $h_{1}$

(b) the performance with $h_{2}$

The size of the filter window is $5 \times 5$. In Fig. 3 (a), $h_{2}=10$, then set $h_{1}$ to $1,5,10,15$, 20. In Fig.3 (b), $h_{1}=10$, then set $h_{2}$ to $1,5,10,15,20$. From Fig.3, we can see that the $h_{2}$ value in the bilateral filter is relatively insensitive to the noise variance compared to the $h_{1}$ value. In our experiments, the values of $h_{1}$ and $h_{2}$ are empirically chosen. We should choose 
the parameter settings that can achieve the best noise reduction results.

Blurring can be difficult to overcome with a BILF. Pixel values on an image are combined with the geometric space information which causes a significant smoothness across boundaries. It is vital to apply more enhanced techniques to deal with blurring.

\section{The proposed method}

For the NLM, the weights are calculated which only use pixel information to measure the similarity between a central region patch and its neighboring patches. For the BILF, the weights use the spatial similarity and the pixel similarity. In this paper, the proposed method is to combine the advantages of the NLM and the BILF with added texture information as weights.

Let $\mathrm{S}$ represent the mean squared deviation (MSD) in patch window (Equation (6)). Each window has an "S". The MSD can differentiate the smooth area and edge of image. In smooth area, the MSD is nearly 0. For edge or spot, the MSD is large. So, it can reflect the patch texture information. $g$ is the average in a patch window. $P$ is the number of pixels in the patch window. $w_{1}\left(x, y, x_{0}, y_{0}\right)$ is the weight value that measures the similarity of the MSD (Equation (7)). $w_{2}\left(x, y, x_{0}, y_{0}\right)$ is the weight value that measures the similarity of spatially close to the current pixel (Equation (8)).

$$
\begin{gathered}
S=\frac{1}{\sqrt{3} p} \sum_{p=0}^{P-1}\left\|f_{k}(x, y)-g_{k}\right\|_{2} f_{k}(x, y) \in \text { PatchWindow } \\
w_{1}\left(x, y, x_{0}, y_{0}\right)=\exp -\left(\frac{\left(S_{x, y}-S_{x_{0}, y_{0}}\right)^{2}}{h_{1}}\right) \\
w_{2}\left(x, y, x_{0}, y_{0}\right)=\exp \left(-\frac{\left(x-x_{0}\right)^{2}+\left(y-y_{0}\right)^{2}}{h_{2}}\right) \\
w\left(x, y, x_{0}, y_{0}\right)=\exp \left(-\frac{\sum_{p \in P}\left\|f_{k}(x+p, y+p)-f_{k}\left(x_{0}+p, y_{0}+p\right)\right\|_{2}^{2}}{h}\right) w_{1}\left(x, y, x_{0}, y_{0}\right) w_{2}\left(x, y, x_{0}, y_{0}\right)
\end{gathered}
$$

We set $h_{1}=100, h_{2}=50, h_{3}=60$. A new weight is computed by Equation (9). The proposed improved non-local means (INLM) algorithm is described as follows.

Input: The noisy image $f_{k}(x, y)$, with size of $H \times W \times 3$

1: Initialize (searching window) $N=5 \times 5$, (patch window) $P=3 \times 3, h_{1}=100$, $h_{2}=50, h_{3}=60$.

2: Use Equation (6) to compute the MSD of each patch window.

3: for $\mathrm{x}=1$ to $\mathrm{H} ; \mathrm{y}=1$ to $\mathrm{W}$

4: for Searching window $\mathrm{n}=1$ to $\mathrm{N}$

5: $\quad$ for PatchWindow $\mathrm{p}=1$ to $\mathrm{P}$

6: $\quad$ Use Equation (2) to compute w(u,v) . 
7: $\quad$ end for

8: end for

9: $\quad$ Compute $w_{1}\left(x, y, x_{0}, y_{0}\right), w_{2}\left(x, y, x_{0}, y_{0}\right)$

10: $\quad$ Use Equation (9) to compute w.

11: Use Equation (1) to get the filtering value of position (x,y).

12: end for

Output: The restored image $g_{k}(x, y)$

\section{Experiments and results}

An extensive set of experiments have been conducted in this research. The experiments aim at evaluating and analyzing the behavior of the proposed image filtering technique in comparing with other techniques. Most of the filtering images in other works are gray images, however our method mainly focuses on color images. We use some similar images as in $[14,16]$, for example the "onion" image. Other images all come from the same public datasets in Matlab, including "hestain" image with $303 \times 227$, "gantrycrane" image with $400 \times 264$, "onion" image with $198 \times 135$ and "football" image with $320 \times 256$. Fig. 4 shows the original images.

We assess the performances of the algorithm with several popularly used test images using Matlab 7.0. The execution time (in seconds) running on a desktop PC with $2.50 \mathrm{GHz}$ CPU and 4.0GB RAM is measured.

A quantitative comparison was performed between the performances of the proposed denoising algorithm and other existing methods. Peak Signal-to-Noise Ratio (PSNR), normalized mean square error (NMSE), mean squared error (MSE) and structural similarity (SSIM) [11] are adopted to evaluate the performance of different denoising methods. MSE and NMSE are represented in pixels, and PSNR is represented in decibels (dB). SSIM a method for measuring the similarity between two images. These criteria are defined as follows (Equations (10) (11) (12) (13)).

$$
\begin{gathered}
P S N R=10 \lg \frac{3 \times 255^{2}}{\frac{1}{H W} \sum_{x=1}^{H} \sum_{y=1}^{W}\left[g_{k}(x, y)-o_{k}(x, y)\right]^{2}} \\
N M S E=\frac{\sum_{x=1}^{H} \sum_{y=1}^{W}\left\|g_{k}(x, y)-o_{k}(x, y)\right\|_{2}^{2}}{\sum_{x=1}^{H} \sum_{y=1}^{W}\left\|o_{k}(x, y)\right\|_{2}^{2}} \\
\operatorname{MSE}=\frac{1}{3 H W} \sum_{x=1}^{H} \sum_{y=1}^{W}\left\|g_{k}(x, y)-o_{k}(x, y)\right\|_{2}^{2} \\
\operatorname{SSIM}(g, o)=\frac{\left(2 \mu_{g} \mu_{o}+c_{1}\right)\left(2 \sigma_{g o}+c_{2}\right)}{\left(\mu_{g}^{2}+\mu_{o}^{2}+c_{1}\right)\left(\sigma_{g}^{2}+\sigma_{o}^{2}+c_{2}\right)}
\end{gathered}
$$

$(x, y)$ characterizes the sample position, $o_{k}(x, y)$ is the original, desired image and $g_{k}(x, y)$ represents the output of the filter image, where $\mu_{g}$ and $\mu_{o}$ are the average of $\mathrm{g}$ and 
o, respectively. $\sigma_{g}^{2}$ and $\sigma_{o}^{2}$ are the variance of $\mathrm{g}$ and o, respectively. $\sigma_{\mathrm{go}}$ is the covariance of g and o. $c_{1}=\left(k_{1} L\right)^{2}, c_{2}=\left(k_{2} L\right)^{2} . \mathrm{L}$ is the dynamic range of the pixel-values, $k_{1}=0.01$ and $k_{2}=0.03$ by default. To evaluate the performances of noise suppression and edge preservation, the proposed algorithm is compared with the NLM, BILF, a modified switching median filter (MSMF) by Wang et al. [4], FDNLM [20] and GNLMKIM [19]. All the images can be tested in the same way for Gaussian noise and mixture noise. Due to the limitation of the article length, we only present some of the testing results.

\subsection{Gaussian noise}

In this experiment, the color images are corrupted with Gaussian noise with different standard deviations.

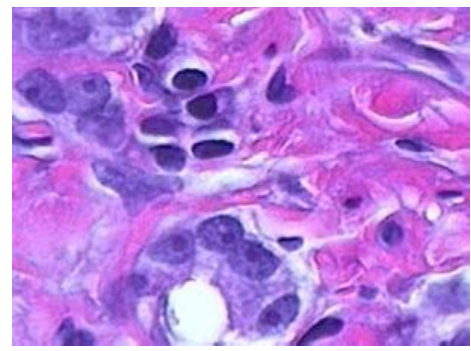

(a)

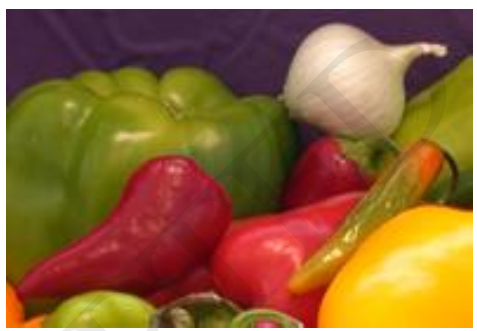

(b)
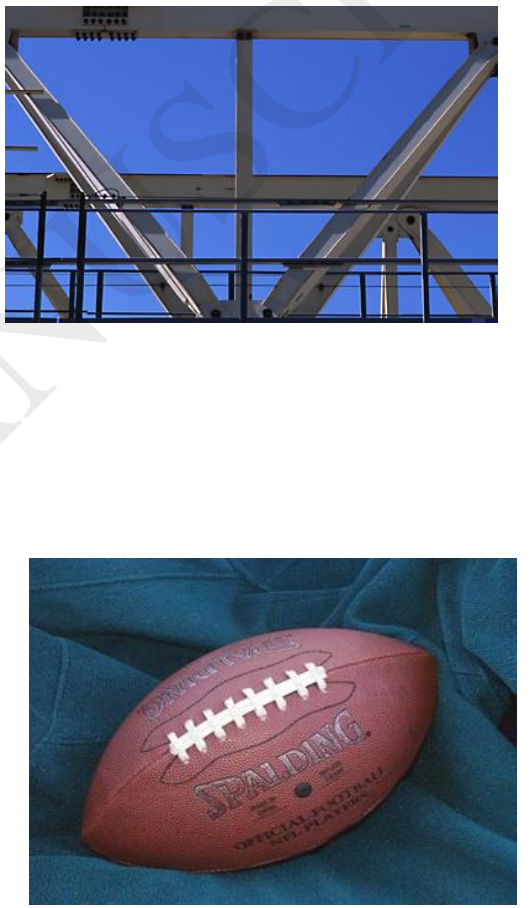

(c)

Fig.4 The original images: (a) "hestain" (b) "gantrycrane" (c) "onion" (d) "football"

Fig.5 shows the filtering outputs from "hestain" image. We add Gaussian noise with a mean of 0 and variance of 0.02 to the original image. In the NLM, with an increasing searching window size, the blurring appears more severe. The computational time is also increasing. The size of the searching window is set to $7 \times 7$, and the size of the patch window is $3 \times 3$. In the BILF, the filtering window is $3 \times 3$. For the INLM, the size of the searching window is $5 \times 5$ and the size of the patch window is $3 \times 3$. In the MSMF, the filtering window is $3 \times 3$. The MSMF can remove the impulse noise effectively, but it produces bad results with Gaussian noise.

The comparison results of the performances are shown in Tables 1-3. The MSMF has a 
lower PSNR and a higher NMSE and MSE. It is demonstrated that the MSMF is excellent for removing the impulse noise, but is not so capable to remove Gaussian noise. The NLM and FDNLM has a longer computational time for "hestain" image. The INLM has a better performance, and its computational time is acceptable.

Fig.6 shows the filtering outputs for the "gantrycrane" image. We add Gaussian noise of mean 0 and variance of 0.03 to the original image. Fig. 7 shows the filtering results in PSNR (dB) with the filters applied to the images.

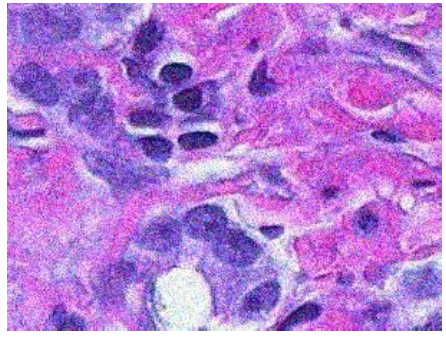

(a)

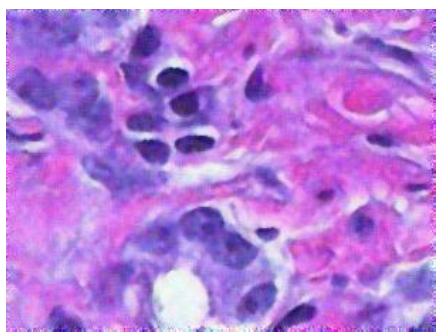

(c)

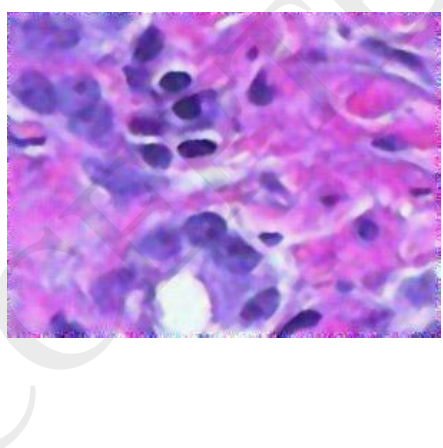

(e)

Fig.5 Performance evaluation: (a) the "hestain" image with Gaussian noise (b) the NLM output (c) the BILF output (d) the MSMF output (e) the FDNLM output (f) the output from the proposed algorithm (b)
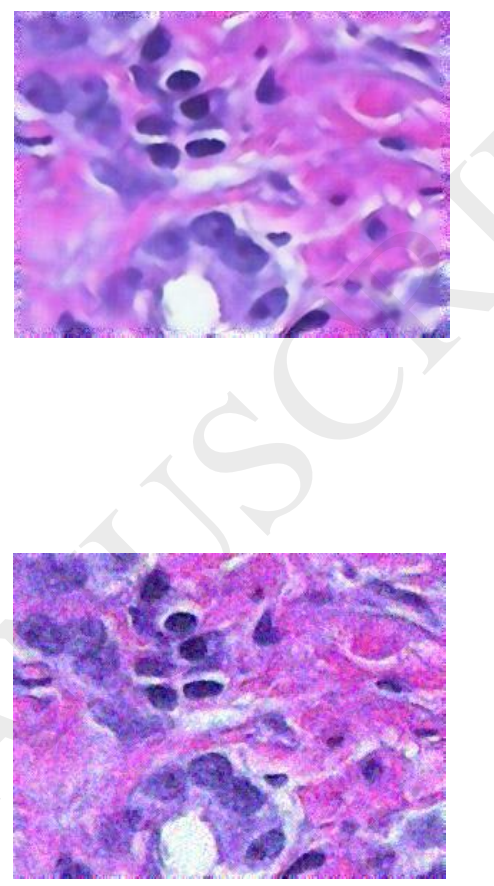

(d)

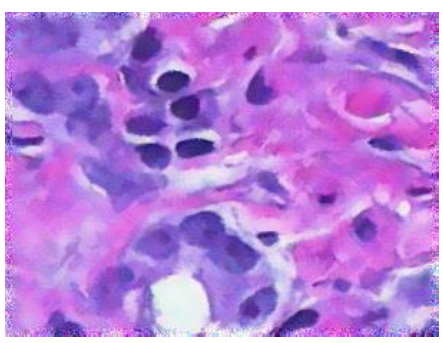

(f) 


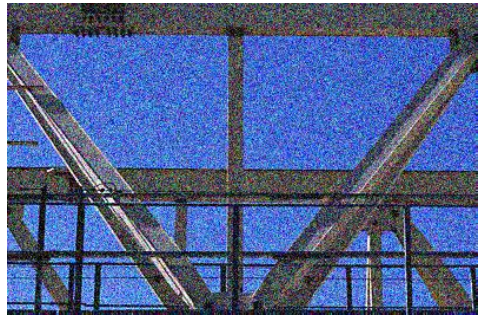

(a)

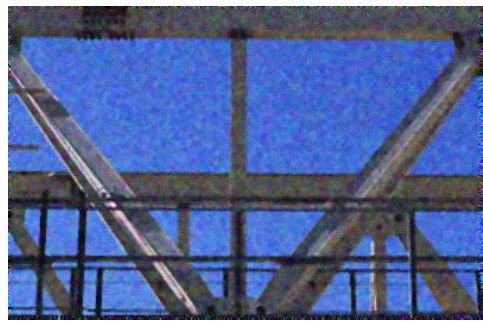

(c)

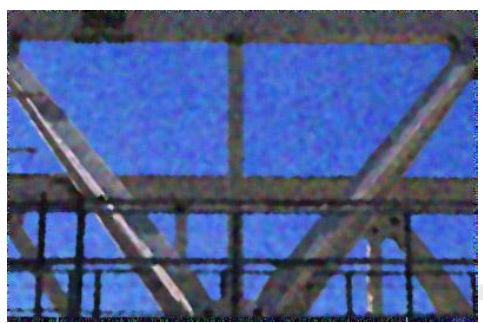

(e)

Fig.6 Performance evaluation: (a) the "gantrycrane" image with Gaussian noise (b) the NLM output (c) the BILF output (d) the MSMF output (e) the FDNLM output (f) the output from the proposed algorithm

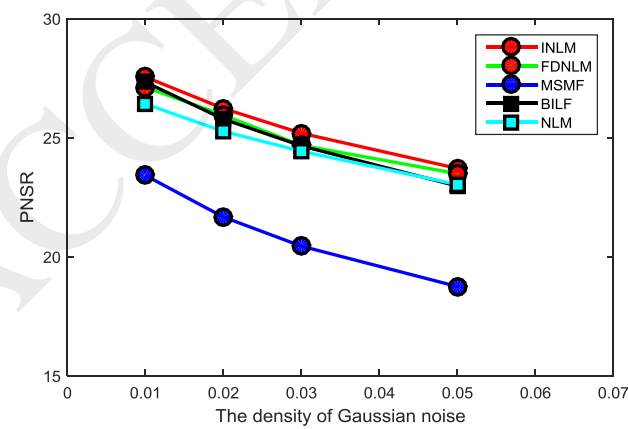

(a)

Fig.7 Filtering results in PSNR (dB) from the different filters applied to the images: (a) PSNR for the "hestain" image (b) PSNR for the "gantrycrane" image

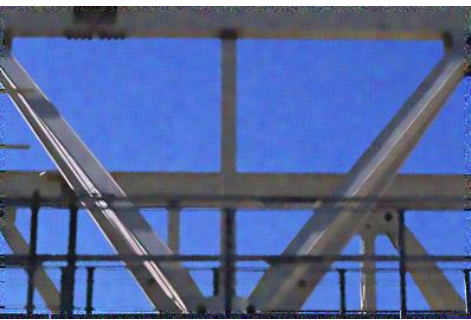

(b)

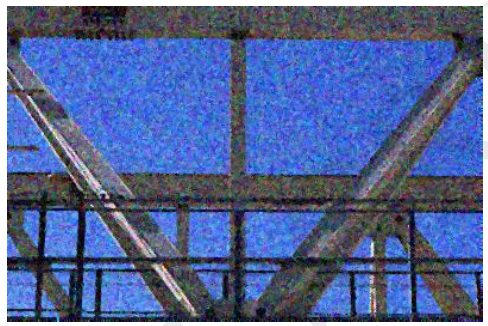

(d)

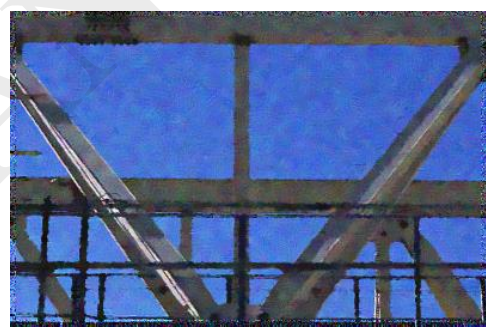

(f)

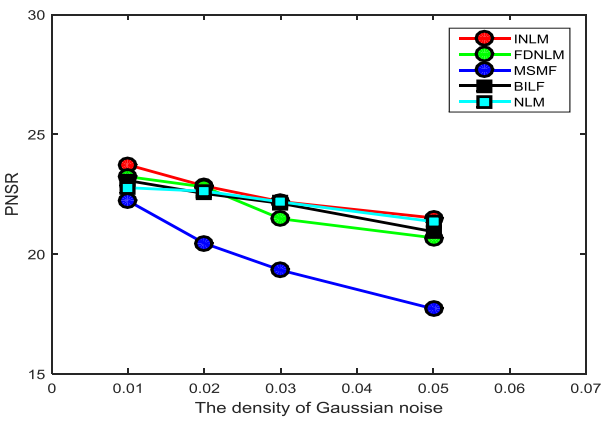

(b) 


\subsection{Mixture noise}

As the impact caused by Gaussian noise and impulse noise on images is different, little research has been reported on removing the mixture of both types of noise. In this paper, the proposed method combines the advantages of the NLM and the BILF, and exhibits good performances in removing the complicated mixtures. The comparison results of the performances are shown in Tables 4-6.

Fig. 8-9 and Fig. 11 show the filtering outputs from different filters for the different images. We added the mixture noise, combining with $3 \%$ impulse noise and Gaussian noise of mean 0 and variance 0.01 , to original images. Fig. 10 shows the filtering results in PSNR (dB) from the filters applied to the images.

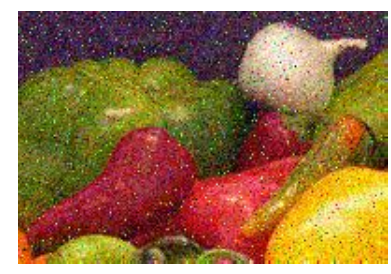

(a)

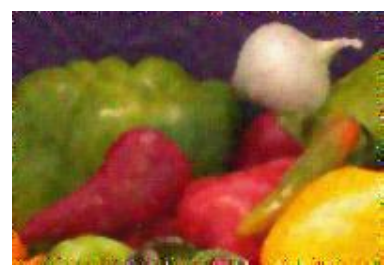

(c)

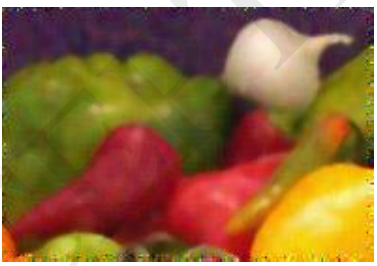

(e)

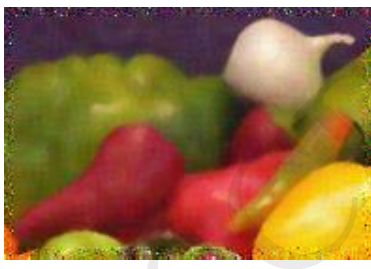

(b)

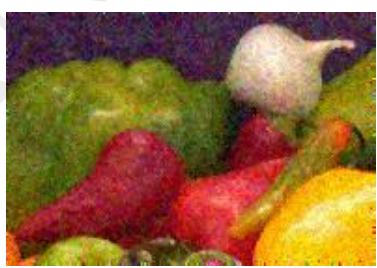

(d)

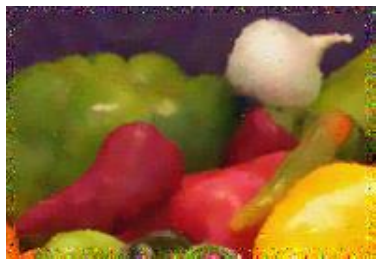

(f)

Fig.8 Performance evaluation: (a) the "onion" image with mixture noise (b) the NLM output (c) the BILF output (d) the MSMF output (e) the FDNLM output (f) the output from the proposed algorithm 


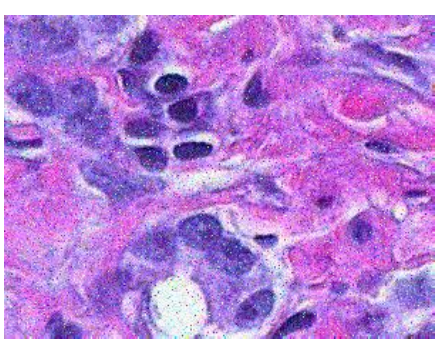

(a)

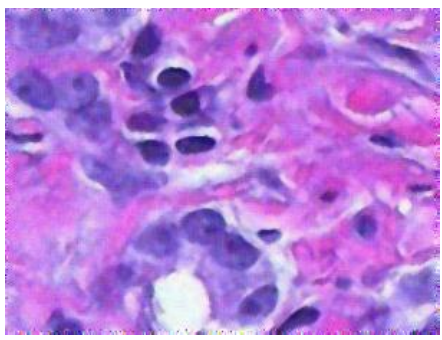

(c)

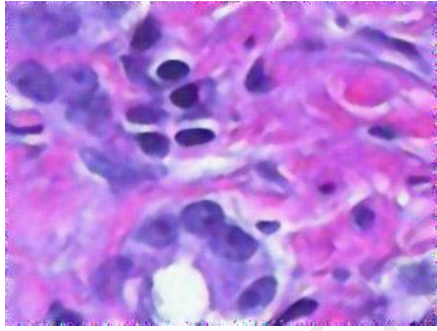

(e)

(f)

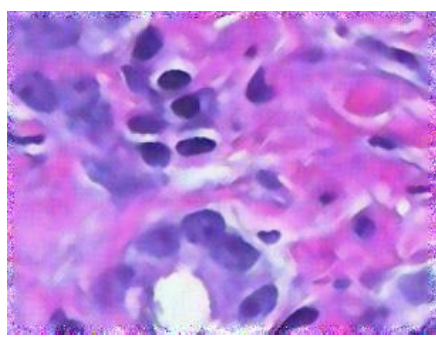

(b)

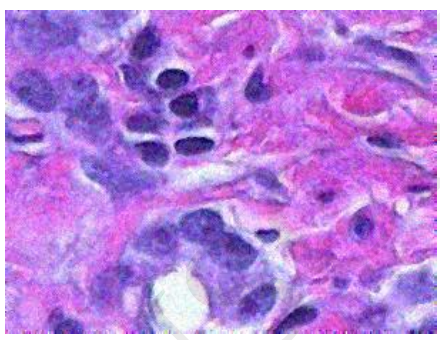

(d)

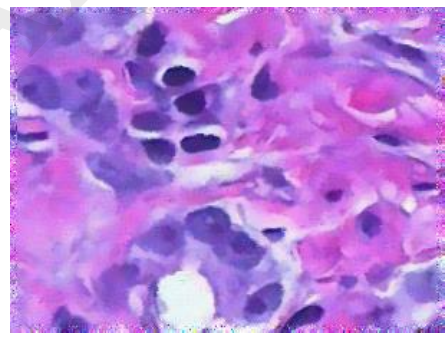

Fig.9 Performance evaluation: (a) the "hestain" image with mixture noise (b) the NLM output (c) the BILF output (d) the MSMF output (e) the FDNLM output (f) the output from the proposed algorithm

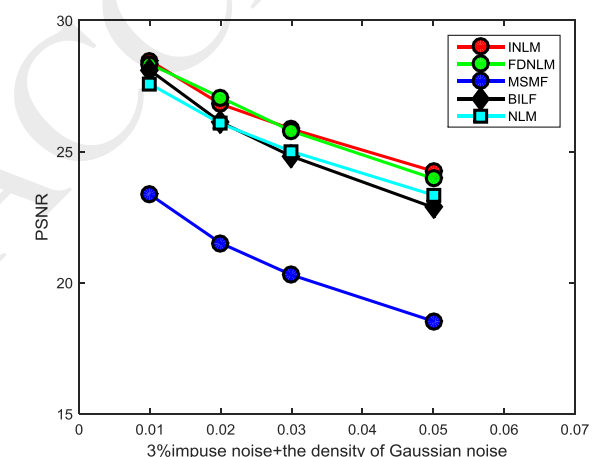

(a)

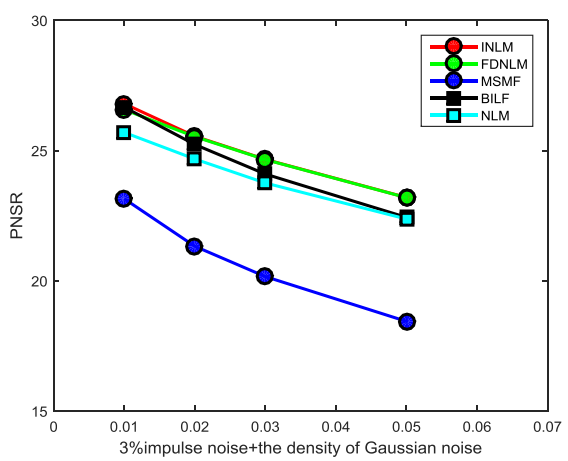

(b) the "onion" image. (b) PSNR for the "hestain" image 


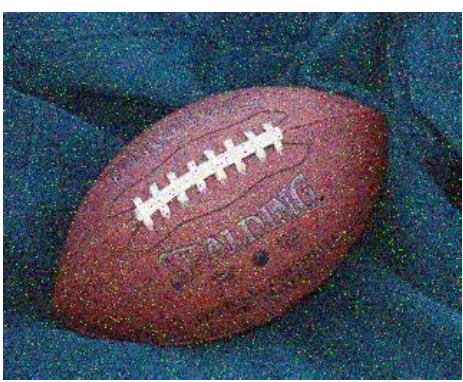

(a)

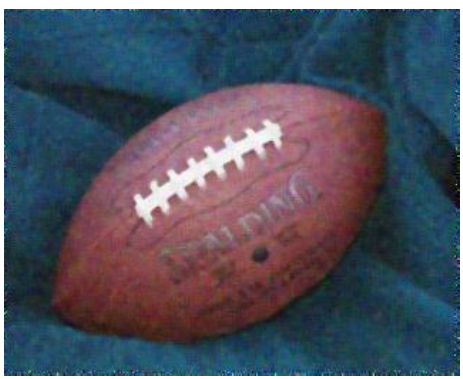

(c)

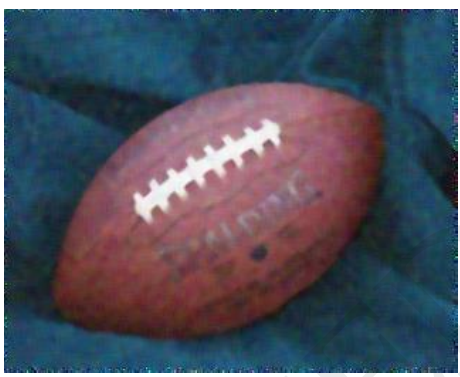

(e)

Fig.11 Performance evaluation: (a) the "football" image with mixture noise (b) the NLM output (c) the BILF output (d) the MSMF output (e) the FDNLM output (f) the output from the proposed algorithm

\subsection{Discussion}

The experimental results from Fig.7 and Fig.10 can be summarized. The NLM filter is better for dealing with Gaussian noise. When the level of Gaussian noise increases, the NLM performs better than the BILF. From Fig.7 and Fig.10, the density level of Gaussian noise increases from 0.01 to 0.03 , and the NLM results a higher PSNR than that of the BILF. The experiments show that the MSMF cannot remove Gaussian noise effectively, however, the PSNR from the MSMF filter is $41.8259 \mathrm{~dB}$ when $3 \%$ of impulse noise is added to the original image. For FDNLM, the filter works better for lower Gaussian noise. From Fig. 2, one can see that a bigger searching window contains more pixels and has higher statistical reliability but increases the computation time. In case of NLM, a big searching window would blur the images more. In this paper, the size of the searching window is chosen empirically. 
Tables 1-3 are the performance comparisons for the "hestain", "gantrycrane" and "onion" images with added Gaussian noise. From Table 1, we can see that our method has better performance when the variance of Gaussian noise is $0.01,0.02$ and 0.05 . When the variance of Gaussian noise is 0.03 , our method has a similar performance as GNLMKIM. In computer time, except for BILF, our method is faster than others. Tables 2-3 have the same conclusions. Tables 4-6 are the performance comparisons for the "onion", "hestain" and "football" images with added mixture noise. From Table 4, we can see that our method has the best performance. The computer time is also acceptable. From Table 5, our method has better performance when the density of noise is low. When the density of noise is high, our method has a similar performance as GNLMKIM. From Tables 1-6, one can see that the computational time is longer for the FDNLM and NLM. The size of the searching window is $7 \times 7$. The NLM needs to compute the similarities between a central region patch and its neighboring patches in a search window and thus consumes more time. In the proposed method INLM, the size of the searching window is set to $5 \times 5$ to reduce the computational time, but it is still the best with the highest PSNR and SSIM, lowest MSE and NMSE. For removing the mixture noise, the performance of INLM is almost as good as FDNLM, but the computation time is significantly less. For example, in Table 4 the computational time of INLM is 1-2 seconds whereas FDNLM is $10-11$ seconds; in Table 5 the computational time of INLM is 3-4 seconds whereas FDNLM is 26-29 seconds. In these cases, the real-time performance of the proposed method is significantly better.

Table 1 Performance comparison for the "hestain" image with added Gaussian noise

\begin{tabular}{|c|c|c|c|c|c|c|c|c|c|c|}
\hline \multirow{2}{*}{$\begin{array}{l}\text { Noise } \\
\text { Algorithms }\end{array}$} & \multicolumn{5}{|c|}{ Gaussian noise of mean 0 and variance 0.01} & \multicolumn{5}{|c|}{ Gaussian noise of mean 0 and variance 0.02} \\
\hline & PSNR & NMSE & MSE & SSIM & Time(s) & PSNR & NMSE & MSE & SSIM & Time(s) \\
\hline NLM & 26.4173 & 0.0036 & 148.3717 & 0.6699 & 4.772590 & 25.2676 & 0.0058 & 193.3411 & 0.6690 & 5.023923 \\
\hline BILF & 27.3346 & 0.0044 & 120.1204 & 0.6100 & 1.622203 & 25.7838 & 0.0051 & 171.6705 & 0.5611 & 1.684648 \\
\hline MSMF[4] & 23.4167 & 0.0134 & 296.0820 & 0.5736 & 3.307964 & 21.6688 & 0.0132 & 442.7938 & 0.4815 & 3.466207 \\
\hline FDNLM[20] & 27.1223 & 0.0050 & 138.7452 & 0.7104 & 27.74955 & 25.9487 & 0.0048 & 161.2211 & 0.6695 & 27.8416 \\
\hline $\begin{array}{l}\text { GNLM } \\
\text { KIM[19] }\end{array}$ & 26.3920 & 0.0044 & 149.4430 & 0.6774 & 21.17379 & 25.9500 & 0.0049 & 165.2258 & 0.6759 & 21.19342 \\
\hline INLM & 27.5517 & 0.0028 & 114.2647 & 0.7705 & 3.039173 & 26.2277 & 0.0046 & 154.9926 & 0.7204 & 3.482848 \\
\hline Noise & \multicolumn{5}{|c|}{ Gaussian noise of mean 0 and variance 0.03} & \multicolumn{5}{|c|}{ Gaussian noise of mean 0 and variance 0.05} \\
\hline Algorithms & PSNR & NMSE & MSE & SSIM & Time(s) & PSNR & NMSE & MSE & SSIM & Time(s) \\
\hline NLM & 24.4336 & 0.0070 & 234.2737 & 0.5273 & 5.063107 & 23.0335 & 0.0096 & 323.3958 & 0.5849 & 5.031775 \\
\hline BILF & 24.6571 & 0.0066 & 222.5202 & 0.6089 & 1.701433 & 22.9716 & 0.0098 & 328.0370 & 0.4856 & 1.761296 \\
\hline MSMF[4] & 20.4470 & 0.0174 & 586.6493 & 0.4225 & 3.587132 & 18.7509 & 0.0258 & 866.9503 & 0.3451 & 3.724869 \\
\hline FDNLM[20] & 24.6820 & 0.0066 & 221.2491 & 0.6325 & 26.98131 & 23.4907 & 0.0087 & 291.0758 & 0.6243 & 27.16453 \\
\hline $\begin{array}{l}\text { GNLMKIM[ } \\
\text { 19] }\end{array}$ & 25.2857 & 0.0057 & 192.5369 & 0.6751 & 21.68529 & 23.6346 & 0.0084 & 281.5897 & 0.6732 & 21.09615 \\
\hline
\end{tabular}




\begin{tabular}{l|l|l|l|l|l|l|l|l|l|l}
\hline INLM & 25.1892 & 0.0059 & 196.8633 & 0.6870 & 3.511491 & $\mathbf{2 3 . 7 1 2 8}$ & $\mathbf{0 . 0 0 8 2}$ & $\mathbf{2 7 6 . 5 6 8 1}$ & $\mathbf{0 . 6 6 2 1}$ & 3.494458 \\
\hline
\end{tabular}

Table 2 Performance comparison for "gantrycrane" with added Gaussian noise

\begin{tabular}{|c|c|c|c|c|c|c|c|c|c|c|}
\hline \multirow{2}{*}{$\begin{array}{l}\text { Noise } \\
\text { Algorithms }\end{array}$} & \multicolumn{5}{|c|}{ Gaussian noise of mean 0 and variance 0.01} & \multicolumn{5}{|c|}{ Gaussian noise of mean 0 and variance 0.02} \\
\hline & PSNR & NMSE & MSE & SSIM & Time(s) & PSNR & NMSE & MSE & SSIM & Time(s) \\
\hline NLM & $\begin{array}{l}22.773 \\
2\end{array}$ & 0.0232 & 343.3695 & 0.5370 & 7.957388 & 22.6303 & 0.0239 & 354.8549 & 0.6811 & 7.902535 \\
\hline BILF & $\begin{array}{l}23.070 \\
9\end{array}$ & 0.0216 & 320.0208 & 0.7098 & 2.721877 & 22.5319 & 0.0245 & 362.9832 & 0.4775 & 2.681040 \\
\hline MSMF[4] & $\begin{array}{l}22.224 \\
6\end{array}$ & 0.0263 & 389.5989 & 0.4008 & 5.534327 & 20.4515 & 0.0395 & 586.0496 & 0.3210 & 5.494203 \\
\hline FDNLM[20] & $\begin{array}{l}23.235 \\
1\end{array}$ & 0.0200 & 290.6636 & 0.7167 & 41.66524 & 22.8056 & 0.0215 & 340.2046 & 0.6942 & 41.78999 \\
\hline $\begin{array}{l}\text { GNLMKIM[ } \\
19]\end{array}$ & $\begin{array}{l}22.990 \\
3\end{array}$ & 0.0220 & 326.6233 & 0.7083 & 37.05799 & 22.7258 & 0.0234 & 347.1334 & 0.6935 & 34.51526 \\
\hline INLM & $\begin{array}{l}23.727 \\
5\end{array}$ & 0.0186 & 275.6294 & 0.7486 & 5.160320 & 22.8462 & 0.0228 & 337.6415 & 0.7055 & 5.574742 \\
\hline Noise & \multicolumn{5}{|c|}{ Gaussian noise of mean 0 and variance 0.03} & \multicolumn{5}{|c|}{ Gaussian noise of mean 0 and variance 0.05} \\
\hline Algorithms & PSNR & NMSE & MSE & SSIM & Time(s) & PSNR & NMSE & MSE & SSIM & Time(s) \\
\hline NLM & $\begin{array}{l}22.182 \\
4\end{array}$ & 0.0265 & 393.4069 & 0.6174 & 7.886772 & 21.3588 & 0.0321 & 475.5556 & 0.5381 & 7.957532 \\
\hline BILF & $\begin{array}{l}22.122 \\
8\end{array}$ & 0.0269 & 398.8403 & 0.5860 & 2.669104 & 20.9397 & 0.0353 & 523.7317 & 0.3475 & 2.695254 \\
\hline MSMF[4] & $\begin{array}{l}19.332 \\
9\end{array}$ & 0.0511 & 758.2119 & 0.2771 & 5.737081 & 17.7217 & 0.0741 & $1.098 \mathrm{e}+003$ & 0.2148 & 5.998339 \\
\hline FDNLM[20] & $\begin{array}{l}21.477 \\
8\end{array}$ & 0.0312 & 462.6971 & 0.4181 & 42.32771 & 20.6845 & 0.0375 & 555.4303 & 0.4894 & 41.60379 \\
\hline $\begin{array}{l}\text { GNLMKIM[ } \\
19]\end{array}$ & $\begin{array}{l}22.349 \\
3\end{array}$ & 0.0255 & 378.5744 & 0.7016 & 34.46457 & 21.3110 & 0.0324 & 480.8218 & 0.6910 & 33.95962 \\
\hline INLM & $\begin{array}{l}22.196 \\
1\end{array}$ & 0.0265 & 392.1644 & 0.7074 & 5.473537 & 21.5148 & 0.0309 & 458.7793 & 0.6982 & 5.515224 \\
\hline
\end{tabular}

Table 3 Performance comparison for the "onion" image with added Gaussian noise

\begin{tabular}{l|l|l|l|l|l|l|l|l|l|l}
\hline \multirow{2}{*}{$\begin{array}{l}\text { Noise } \\
\text { Algorithms }\end{array}$} & \multicolumn{4}{|l}{ Gaussian noise of mean 0 and variance 0.01} & \multicolumn{3}{l}{ Gaussian noise of mean 0 and variance 0.02} \\
\cline { 2 - 11 } & PSNR & NMSE & MSE & SSIM & Time(s) & PSNR & NMSE & MSE & SSIM & Time(s) \\
\hline NLM & 28.2983 & 0.0074 & 96.2175 & 0.7940 & 9.054051 & 27.5160 & 0.0088 & 115.2087 & 0.7946 & 9.140724 \\
& & & & & & & & & & \\
\hline BILF & 26.3733 & 0.0115 & 149.8818 & 0.6715 & $\mathbf{7 . 0 2 1 6 9 0}$ & 25.4059 & 0.0144 & 187.2800 & 0.6239 & $\mathbf{7 . 0 8 3 7 9 6}$ \\
\hline FDNLM[20] & 28.4857 & 0.0071 & 92.1532 & 0.8058 & 10.26517 & 27.2751 & 0.0093 & 121.7770 & 0.7659 & 10.186742 \\
\hline
\end{tabular}




\begin{tabular}{|c|c|c|c|c|c|c|c|c|c|c|}
\hline $\begin{array}{l}\text { GNLMKIM[ } \\
19]\end{array}$ & 28.3725 & 0.0073 & 94.5871 & 0.7930 & 7.741945 & 27.5422 & 0.0088 & 114.5151 & 0.7936 & 8.212901 \\
\hline INLM & 28.4602 & 0.0071 & 92.6955 & 0.7948 & 9.186389 & 27.6890 & 0.0085 & 110.7083 & 0.7951 & 9.161111 \\
\hline Noise & \multicolumn{5}{|c|}{ Gaussian noise of mean 0 and variance 0.03} & \multicolumn{5}{|c|}{ Gaussian noise of mean 0 and variance 0.05} \\
\hline Algorithms & PSNR & NMSE & MSE & SSIM & Time(s) & PSNR & NMSE & MSE & SSIM & Time(s) \\
\hline NLM & 26.5427 & 0.0111 & 144.1487 & 0.7952 & 9.21583 & 24.2627 & 0.0187 & 243.6732 & 0.7842 & 9.121792 \\
\hline BILF & 24.3940 & 0.0181 & 236.4169 & 0.5779 & 7.221277 & 23.0197 & 0.0249 & 324.4218 & 0.5115 & 7.143101 \\
\hline FDNLM[20] & 26.1106 & 0.0122 & 159.2293 & 0.7156 & 10.17484 & 24.5156 & 0.0176 & 229.8919 & 0.6398 & 10.448825 \\
\hline $\begin{array}{l}\text { GNLMKIM[ } \\
\text { 19] }\end{array}$ & 26.5991 & 0.0109 & 142.2871 & 0.7913 & 7.755470 & 24.2700 & 0.0186 & 243.2626 & 0.7906 & 7.798624 \\
\hline INLM & 26.6541 & 0.0108 & 140.4966 & 0.7970 & 9.131547 & 24.3855 & 0.0182 & 236.8788 & 0.7928 & 9.167428 \\
\hline
\end{tabular}

Table 4 Performance comparison for "onion" image with added mixture noise

\begin{tabular}{|c|c|c|c|c|c|c|c|c|c|c|}
\hline \multirow{2}{*}{$\begin{array}{l}\text { Noise } \\
\text { Algorithms }\end{array}$} & \multicolumn{5}{|c|}{$\begin{array}{l}3 \% \text { impulsive noise plus Gaussian noise of mean } 0 \text { and } \\
\text { variance } 0.01\end{array}$} & \multicolumn{5}{|c|}{$\begin{array}{l}3 \% \text { impulsive noise plus Gaussian noise of mean } 0 \text { and } \\
\text { variance } 0.02\end{array}$} \\
\hline & PSNR & NMSE & MSE & SSIM & Time(s) & PSNR & NMSE & MSE & SSIM & Time(s) \\
\hline NLM & 27.5767 & 0.0087 & 113.6094 & 0.7394 & 1.817586 & 26.0671 & 0.0123 & 160.8327 & 0.7463 & 1.792076 \\
\hline BILF & 28.1025 & 0.0077 & 100.6532 & 0.6512 & 0.629034 & 26.1226 & 0.0122 & 158.7879 & 0.6002 & 0.614919 \\
\hline MSMF[4] & 23.3636 & 0.0230 & 300.0121 & 0.4489 & 1.270196 & 21.5076 & 0.0352 & 459.5377 & 0.3733 & 1.298394 \\
\hline FDNLM[20] & 28.3366 & 0.0073 & 95.3713 & 0.8054 & 10.23720 & 27.0634 & 0.0098 & 127.8613 & 0.7541 & 10.25267 \\
\hline $\begin{array}{l}\text { GNLMKIM[ } \\
\text { 19] }\end{array}$ & 27.2660 & 0.0092 & 120.5987 & 0.7414 & 7.860038 & 26.5397 & 0.0111 & 144.2467 & 0.7516 & 8.092862 \\
\hline INLM & 28.4632 & 0.0071 & 92.6313 & 0.8071 & 1.246791 & 26.8081 & 0.0104 & 135.6030 & 0.7491 & 1.230364 \\
\hline Noise & \multicolumn{5}{|c|}{$\begin{array}{l}3 \% \text { impulsive noise plus Gaussian noise of mean } 0 \text { and } \\
\text { variance } 0.03\end{array}$} & \multicolumn{5}{|c|}{$\begin{array}{l}3 \% \text { impulsive noise plus Gaussian noise of mean } 0 \text { and } \\
\text { variance } 0.05\end{array}$} \\
\hline Algorithms & PSNR & NMSE & MSE & SSIM & Time(s) & PSNR & NMSE & MSE & SSIM & Time(s) \\
\hline NLM & 25.0052 & 0.0157 & 205.3803 & 0.6941 & 1.772425 & 23.3445 & 0.0231 & 301.0475 & 0.6070 & 1.793721 \\
\hline BILF & 24.8193 & 0.0164 & 214.3643 & 0.5472 & 0.602130 & 22.8719 & 0.0257 & 335.6536 & 0.4917 & 0.602628 \\
\hline MSMF[4] & 20.2975 & 0.0466 & 607.1945 & 0.3169 & 1.317996 & 18.5359 & 0.0698 & 910.9470 & 0.2581 & 1.362528 \\
\hline FDNLM[20] & 25.7784 & 0.0132 & 171.8861 & 0.6987 & 10.29378 & 23.9750 & 0.0200 & 260.3652 & 0.6288 & 10.30350 \\
\hline $\begin{array}{l}\text { GNLMKIM[ } \\
\text { 19] }\end{array}$ & 25.7804 & 0.0132 & 171.8083 & 0.7027 & 7.900953 & 23.6462 & 0.0215 & 280.8426 & 0.7320 & 7.892369 \\
\hline INLM & 25.8593 & 0.0129 & 168.7146 & 0.7475 & 1.226907 & 24.2557 & 0.0187 & 244.0673 & 0.7421 & 1.216469 \\
\hline
\end{tabular}

Table 5 Performance comparison for "hestain" image with added mixture noise

\begin{tabular}{l|l|l|l|l|l|l|l|l|l|l|l}
\hline Noise & \multicolumn{3}{l}{$\begin{array}{l}3 \% \text { impulsive noise plus Gaussian noise of mean } 0 \text { and } \\
\text { variance 0.01 }\end{array}$} & $\begin{array}{l}3 \% \text { impulsive noise plus Gaussian noise of mean } 0 \text { and } \\
\text { variance 0.02 }\end{array}$ \\
\cline { 2 - 7 } Algorithms & PSNR & NMSE & MSE & SSIM & Time(s) & PSNR & NMSE & MSE & SSIM & Time(s) \\
\hline
\end{tabular}




\begin{tabular}{|c|c|c|c|c|c|c|c|c|c|c|}
\hline NLM & 25.7019 & 0.0052 & 174.9409 & 0.6451 & 4.879495 & 24.6812 & 0.0066 & 221.2906 & 0.6435 & 4.875592 \\
\hline BILF & 26.6713 & 0.0042 & 139.9427 & 0.7131 & 1.666603 & 25.2382 & 0.0058 & 194.6517 & 0.5526 & 1.634524 \\
\hline MSMF[4] & 23.1546 & 0.0094 & 314.4999 & 0.5594 & 3.160416 & 21.3313 & 0.0142 & 478.5715 & 0.4678 & 3.414356 \\
\hline FDNLM[20] & 26.5767 & 0.0043 & 143.0243 & 0.7071 & 26.89121 & 25.5378 & 0.0054 & 181.6759 & 0.6718 & 26.930342 \\
\hline $\begin{array}{l}\text { GNLMKIM[ } \\
\text { 19] }\end{array}$ & 25.7610 & 0.0051 & 172.5760 & 0.6495 & 22.18726 & 25.4542 & 0.0055 & 185.2101 & 0.6445 & 22.03886 \\
\hline INLM & 26.8047 & 0.0040 & 135.7089 & 0.7369 & 3.396940 & 25.5564 & 0.0054 & 180.9011 & 0.6932 & 3.378729 \\
\hline Noise & \multicolumn{5}{|c|}{$\begin{array}{l}3 \% \text { impulsive noise plus Gaussian noise of mean } 0 \text { and } \\
\text { variance } 0.03\end{array}$} & \multicolumn{5}{|c|}{$\begin{array}{l}3 \% \text { impulsive noise plus Gaussian noise of mean } 0 \text { and } \\
\text { variance } 0.05\end{array}$} \\
\hline Algorithms & PSNR & NMSE & MSE & SSIM & Time(s) & PSNR & NMSE & MSE & SSIM & Time(s) \\
\hline NLM & 23.7601 & 0.0081 & 273.5718 & 0.6397 & 4.808518 & 22.3767 & 0.0112 & 376.1927 & 0.5725 & 4.799906 \\
\hline BILF & 24.1028 & 0.0075 & 252.8125 & 0.5184 & 1.625290 & 22.4397 & 0.0110 & 370.7732 & 0.4708 & 1.617609 \\
\hline MSMF[4] & 20.1664 & 0.0186 & 625.8034 & 0.4093 & 3.579840 & 18.4472 & 0.0277 & 929.7420 & 0.3297 & 3.702528 \\
\hline FDNLM[20] & 24.6582 & 0.0066 & 222.4624 & 0.6339 & 28.03796 & 23.1979 & 0.0093 & 311.3789 & 0.5974 & 27.05902 \\
\hline $\begin{array}{l}\text { GNLMKIM[ } \\
\text { 19] }\end{array}$ & 24.9390 & 0.0062 & 208.5357 & 0.6468 & 22.29054 & 23.5406 & 0.0086 & 287.7534 & 0.6415 & 22.02678 \\
\hline INLM & 24.6662 & 0.0066 & 222.0538 & 0.6577 & 3.343473 & 23.2033 & 0.0092 & 310.9955 & 0.6360 & 3.316730 \\
\hline
\end{tabular}

Table 6 Performance comparison for "football" image with added mixture noise

\begin{tabular}{|c|c|c|c|c|c|c|c|c|}
\hline Noise & \multicolumn{2}{|c|}{$\begin{array}{l}3 \% \text { impulse noise plus } \\
\text { Gaussian noise of mean } 0 \\
\text { and variance } 0.01\end{array}$} & \multicolumn{2}{|c|}{$\begin{array}{l}3 \% \text { impulse noise plus } \\
\text { Gaussian noise of mean } 0 \\
\text { and variance } 0.02\end{array}$} & \multicolumn{2}{|c|}{$\begin{array}{l}3 \% \text { impulse noise plus } \\
\text { Gaussian noise of mean } 0 \\
\text { and variance } 0.03\end{array}$} & \multicolumn{2}{|c|}{$\begin{array}{l}3 \% \text { impulse noise plus } \\
\text { Gaussian noise of mean } 0 \\
\text { and variance } 0.05\end{array}$} \\
\hline Algorithms & PSNR & MSE & PSNR & MSE & PSNR & MSE & PSNR & MSE \\
\hline NLM & 26.4734 & 146.4660 & 25.3254 & 190.7840 & 24.3672 & 237.8836 & 22.9200 & 331.9568 \\
\hline BILF & 26.9868 & 130.1354 & 25.5218 & 182.3482 & 24.3758 & 237.2636 & 22.7149 & 348.0113 \\
\hline MSMF[4] & 25.4324 & 186.1385 & 24.5474 & 228.2109 & 23.7263 & 275.7078 & 22.5543 & 361.1204 \\
\hline FDNLM[20] & 27.2612 & 122.1685 & 26.3707 & 149.9732 & 25.4284 & 186.3119 & 24.0130 & 258.0945 \\
\hline $\begin{array}{l}\text { GNLMKIM[ } \\
\text { 19] }\end{array}$ & 27.1474 & 125.4113 & 26.3815 & 149.5983 & 25.4989 & 183.3113 & 23.4054 & 296.8518 \\
\hline INLM & 28.0196 & 102.5925 & 27.3508 & 119.6748 & 26.7043 & 138.8826 & 25.4886 & 183.7496 \\
\hline
\end{tabular}

\subsection{Other works}

We also test other recent methods to compare with the proposed method, for example [14,19,37]. The GNLMKIM proposed by Sun et al. [19] utilizes a two-step alternation to get filtering result and has three exponential kernel parameters. In our test, $\mathrm{h} 1$ is set to $10, \mathrm{~h} 2$ is set to 15, and Pi is set to 100. For example, in Table 1 the performance of the proposed method is compared with GNLMKIM as well as other methods using the "hestain" image. With lower 
Gaussian noise, the performance of GNLMKLM is not very good. When the Gaussian noise increases, GNLMKLM performs slightly better but still not as good as the proposed method, and the computation time is much longer than the proposed method. Fig. 12 shows the filtering outputs for the "hestian" image with Gaussian noise. The filtering output of GNLMKLM looks similar as the proposed but by evaluating PNSR, NMSE, MSE and SSIM the proposed is still better in overall. In Table 4, the performance is compared using the "onion" image with mixture noise, and the performance of the GNLMKIM is worse than the proposed. Fig.13 shows the filtering outputs for the "onion" image. Fig.13 (b), (e) is the output of GNLMKLM. Fig.13 (c), (f) is the output of the proposed method. Although Fig.13(c), (f) seem to have more noise, the proposed method can better preserve fine details. To show its performance, Fig.14 shows the zoom-in of image in Fig.13. We can see that GNLMKIM removes some edges and fine details, and makes the image blurred. The proposed method can preserve more details. In addition, the "lion" image that comes from Google "things" dataset [36] is also tested and the performance is compared in Table 7. Fig.15 shows the filtering outputs for the "lion" image. For the "onion" and "lion" images with Gaussian noise, even though the proposed method is slightly slower than GNLMKIM, all the other performances PNSR, NMSE, MSE and SSIM are better.

According to another method by $\mathrm{Li}$ and Suen [14], it applies grey relation to non-local means and make the solution simpler and faster. However, in our experiments the patch of image need to be reshaped as the sequence, and then compute the min and max to obtain the grey relation. We found that although the method provides good results in practice, it also increases the computation time significantly in processing certain images. For example, for the "gantrycrane" image with added Gaussian noise of mean 0 and variance 0.01 using Gaussian kernel, the computation time of [14] is 113.052712s whereas the proposed method is just 5.160320s. Due to its poor computation time, no further performance test is done.

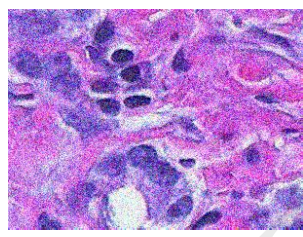

(a)

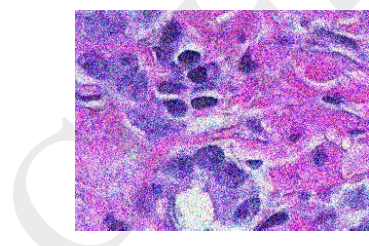

(d)

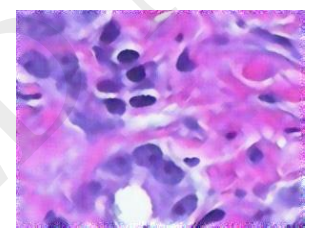

(b)

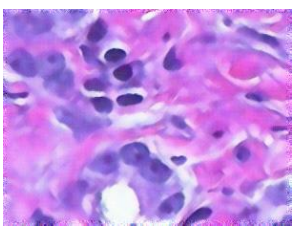

(e)

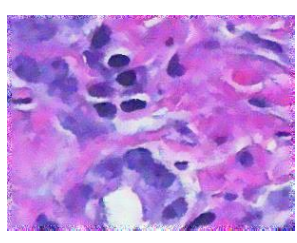

(c)

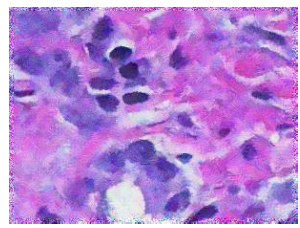

(f)

Fig.12 Performance evaluation: (a) the "hestain" image with Gaussian noise of mean 0 and variance of 0.03 (b) the GNLMKIM output (c) the proposed output (d) the "hestain" image with Gaussian noise of mean 0 and variance of 0.05 (e) the GNLMKIM output (f) the proposed output 


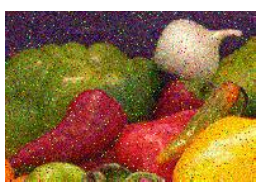

(a)

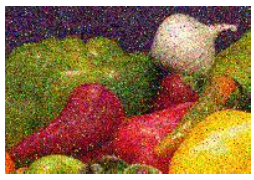

(d)

(e)

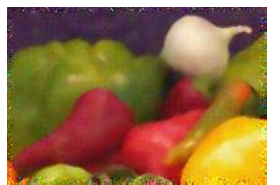

(b)

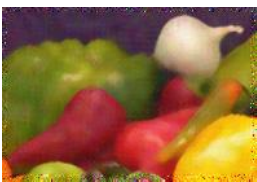

(c)

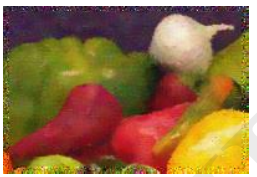

Fig.13 Performance evaluation: (a) the "onion" image with 3\% impulse noise and Gaussian noise of mean 0 and variance of 0.01 (b) the GNLMKIM output (c) the proposed output (d) the "onion" image with 3\% impulse noise and Gaussian noise of mean 0 and variance of 0.02 (e) the GNLMKIM output (f) the proposed output

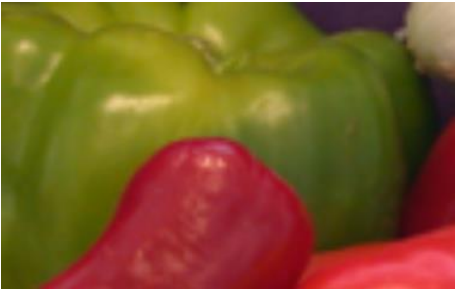

(a)

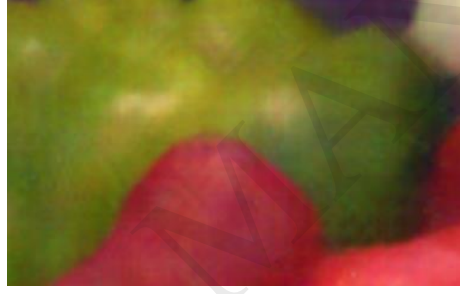

(b)

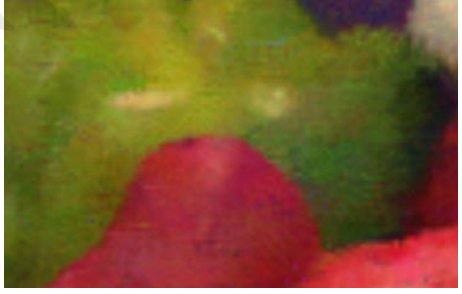

(c)

Fig.14 zoom-in of images in the Fig.13. : (a) zoom-in of the original "onion"(b) zoom-in of the GNLMKIM (c) zoom-in of the proposed

Table 7 Performance comparison for the "lion" image with added Gaussian noise

\begin{tabular}{|c|c|c|c|c|c|c|c|c|c|c|}
\hline \multirow{2}{*}{$\begin{array}{l}\text { Noise } \\
\text { Algorithms }\end{array}$} & \multicolumn{5}{|c|}{ Gaussian noise of mean 0 and variance 0.01} & \multicolumn{5}{|c|}{ Gaussian noise of mean 0 and variance 0.02} \\
\hline & PSNR & NMSE & MSE & SSIM & Time(s) & PSNR & NMSE & MSE & SSIM & Time(s) \\
\hline NLM & 25.4301 & 0.0066 & 186.2408 & 0.7379 & 11.45593 & 25.0926 & 0.0072 & 201.2910 & 0.7368 & 13.521567 \\
\hline BILF & 22.0681 & 0.0144 & 403.8954 & 0.5841 & 9.093267 & 21.4976 & 0.0164 & 460.5993 & 0.5475 & 11.189882 \\
\hline FDNLM[19] & 24.3697 & 0.0085 & 237.7469 & 0.7228 & 12.86418 & 23.6930 & 0.0099 & 277.8306 & 0.6853 & 12.655751 \\
\hline $\begin{array}{l}\text { GNLMKIM[ } \\
18]\end{array}$ & 25.4248 & 0.0066 & 186.4654 & 0.7437 & 9.956551 & 25.0819 & 0.0072 & 201.7876 & 0.7425 & 10.474830 \\
\hline INLM & 25.45381 & 0.0066 & 185.2258 & 0.7483 & 11.73104 & 25.2499 & 0.0069 & 194.1291 & 0.7502 & 12.494737 \\
\hline Noise & \multicolumn{5}{|c|}{ Gaussian noise of mean 0 and variance 0.03} & \multicolumn{5}{|c|}{ Gaussian noise of mean 0 and variance 0.05} \\
\hline
\end{tabular}




\begin{tabular}{l|l|l|l|l|l|l|l|l|l|l}
\hline Algorithms & PSNR & NMSE & MSE & SSIM & Time(s) & PSNR & NMSE & MSE & SSIM & Time(s) \\
\hline NLM & 24.4861 & 0.0082 & 231.4592 & 0.7351 & 11.27403 & 23.1770 & 0.0111 & 312.8854 & 0.7306 & 11.523196 \\
\hline BILF & 21.0864 & 0.0180 & 506.3385 & 0.5135 & $\mathbf{7 . 0 3 5 9 0 0}$ & 20.5120 & 0.0206 & 577.9309 & 0.4710 & $\mathbf{8 . 9 3 2 3 3 3 4}$ \\
\hline FDNLM[19] & 23.1144 & 0.0113 & 317.4252 & 0.6595 & 12.79744 & 22.0551 & 0.0144 & 404.1034 & 0.6019 & 13.178568 \\
\hline $\begin{array}{l}\text { GNLMKIM[ } \\
\text { 18] }\end{array}$ & 24.5859 & 0.0080 & 226.1989 & 0.7353 & 9.709179 & 23.1710 & 0.0111 & 313.3157 & 0.7341 & 10.110937 \\
\hline INLM & $\mathbf{2 4 . 6 7 0 5}$ & $\mathbf{0 . 0 0 7 9}$ & $\mathbf{2 2 1 . 8 3 5 3}$ & $\mathbf{0 . 7 4 2 7}$ & 11.45487 & $\mathbf{2 3 . 3 0 7 8}$ & $\mathbf{0 . 0 1 0 8}$ & $\mathbf{3 0 3 . 5 9 5 7}$ & $\mathbf{0 . 7 4 1 4}$ & 11.873625 \\
\hline
\end{tabular}

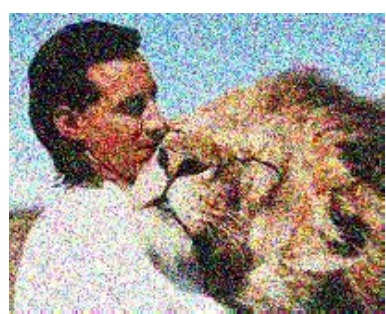

(a)

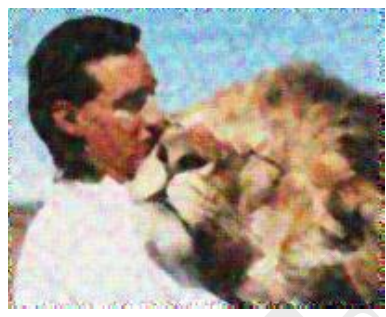

(c)

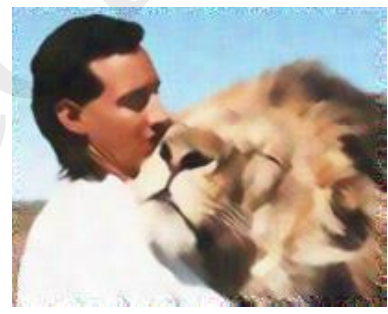

(e)

Fig.15 Performance evaluation: (a) the "lion" image with Gaussian noise of mean 0 and (b)

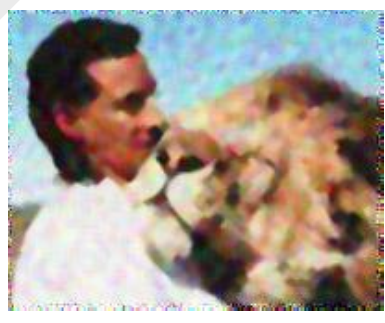

(d)

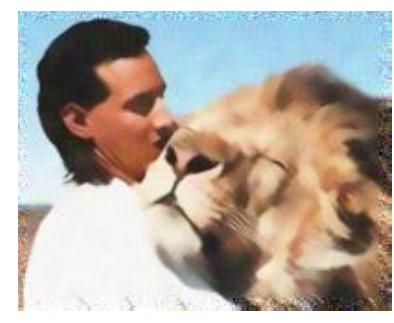

(f) 
variance of 0.05 (b) the NLM output (c) the BILF output (d) the FDNLM output (e) the GNLMKIM output (f) the output from the proposed algorithm

In addition, we also explain the proposed method and the reference paper [37]. The reference paper [37] is a graph-based filter for Gaussian noise. And it computes the Fourier-based dictionaries for each single patch which increase the complexity with the size of dictionary. Dictionary learning has been widely used in restoring Gaussian noise. In our method, it can not only apply Gaussian noise, but also impulse noise or mixture noise. In addition, in our method, we can see that the computer time is faster.

\section{Conclusions}

In this paper, we propose an improved non-local means (INLM) filter for color image denoising. The algorithm combines the advantage of the NLM and the BILF. It calculates a new weight by measuring the spatial similarity, the pixel similarity and the mean of differences. The proposed filter can remove Gaussian noise, impulse noise and the mixture of Gaussian and impulse noise that are added to the original images while preserve the image details very well at different noise ratios. From experimental results, the proposed method has a better denoising performance compared to the several other filters, such as the NLM, the BILF, the MSMF, the FDNLM and the GNLMKIM. In the future work, firstly, we plan to add texture feature to measure similarity of patches. Secondly, we will set up a fast optimization method to solve the model. All attempts can improve the proposed method aiming to remove mixture noise more effectively.

\section{Acknowledgements}

This work is supported by the National Nature Science Fund of China under Grant No. 61601176. 


\section{References}

[1] J. Astola, P. Haavisto, Y. Neuvo, Vector median filters, Proc. IEEE. 78 (1990) 678689. doi:10.1109/5.54807.

[2] C.-C. Kang, W.-J. Wang, Fuzzy reasoning-based directional median filter design, Signal Processing. 89 (2009) 344-351. doi:10.1016/j.sigpro.2008.09.003.

[3] L. Jin, D. Li, A switching vector median filter based on the CIELAB color space for color image restoration, Signal Processing. 87 (2007) 1345-1354.

[4] G. Wang, D. Li, W. Pan, Z. Zang, Modified switching median filter for impulse noise removal, Signal Processing. 90 (2010) 3213-3218.

[5] R. Lukac, K.N. Plataniotis, A.N. Venetsanopoulos, B. Smolka, A Statistically-Switched Adaptive Vector Median Filter, J. Intell. Robot. Syst. 42 (2005) 361-391. doi:10.1007/s10846-005-1730-2.

[6] S. Morillas, V. Gregori, A. Hervás, Fuzzy peer groups for reducing mixed Gaussian-impulse noise from color images, IEEE Trans. Image Process. 18 (2009) 1452-1466.

[7] G. Wang, D. Li, T. Zhao, Adaptive Iteration Filter for Suppression of Impulse Noise in Color Images, Appl. Mech. Mater. 203 (2012) 116-121. doi:10.4028/www.scientific.net/AMM.203.116.

[8] M. Miloslavski, T.-S. Choi, Application of LUM filters with automatic parameter selection to edge detection, in: A.G. Tescher (Ed.), SPIE's Int. Symp. Opt. Sci. Eng. Instrum., International Society for Optics and Photonics, 1998: pp. 865-871. doi:10.1117/12.323156.

[9] L. Liu, C.L.P. Chen, Y. Zhou, X. You, A new weighted mean filter with a two-phase detector for removing impulse noise, Inf. Sci. (Ny). 315 (2015) 1-16. doi:10.1016/j.ins.2015.03.067.

[10] H.-H. Chou, L.-Y. Hsu, A noise-ranking switching filter for images with general fixed-value impulse noises, Signal Processing. $106 \quad$ (2015) 198-208. doi:10.1016/j.sigpro.2014.07.015.

[11] C. Zhang, K. Wang, A switching median-mean filter for removal of high-density impulse noise from digital images, Opt. - Int. J. Light Electron Opt. 126 (2015) 956961. doi:10.1016/j.ijleo.2015.02.085.

[12] G. Wang, Y. Liu, T. Zhao, A quaternion-based switching filter for colour image denoising, Signal Processing. 102 (2014) 216-225. doi:10.1016/j.sigpro.2014.03.027.

[13] A. Buades, B. Coll, J. Morel, A Review of Image Denoising Algorithms, with a New One, Multiscale Model. Simul. 4 (2005) 490-530. doi:10.1137/040616024.

[14] H. Li, C.Y. Suen, A novel Non-local means image denoising method based on grey theory, Pattern Recognit. 49 (2016) 237-248. doi:10.1016/j.patcog.2015.05.028.

[15] Zheng, Y., et al., Adaptively determining regularisation parameters in non-local total variation regularisation for image denoising. Electronics Letters. 51(2) (2015) 144-145.

[16] F. Chen, X. Zeng, M. Wang, Image denoising via local and nonlocal circulant similarity, J. Vis. Commun. Image Represent. 30 (2015) 117-124. doi:10.1016/j.jvcir.2015.03.005. 
[17] H. V Bhujle, S. Chaudhuri, Laplacian based non-local means denoising of MR images with Rician noise., Magn. Reson. Imaging. 31 (2013) 1599-1610. doi:10.1016/j.mri.2013.07.001.

[18] L. Torres, S.J.S. Sant'Anna, C. da Costa Freitas, A.C. Frery, Speckle reduction in polarimetric SAR imagery with stochastic distances and nonlocal means, Pattern Recognit. 47 (2014) 141-157. doi:10.1016/j.patcog.2013.04.001.

[19] Z. Sun, S. Chen, L. Qiao, A general non-local denoising model using multi-kernel-induced measures, Pattern Recognit. 47 (2014) 1751-1763. doi:10.1016/j.patcog.2013.11.003.

[20] G. Wang, H. Zhu, Y. Wang, Fuzzy decision filter for color images denoising, Opt. Int. J. Light Electron Opt. 126 (2015) 2428-2432. doi:10.1016/j.ijleo.2015.06.005.

[21]Liu, Q.,Shu,H.,Sun,B.,Chen B.etal.,Removing Gaussian noise for colour images by quater nion representation and optimisation of weights in non-local means filter. IET Image Pr ocessing, 8(10) (2014) 591-600.

[22] Shim, J., M. Yoon and Y. Lee, Feasibility of newly designed fast non local means(FNLM)-based noise reduction filter for X-ray imaging: A simulation study. Optik, 160(2018) 124-130.

[23] Jomaa, H., et al., Denoising of dynamic PET images using a multi-scale transform and non-local means filter. Biomedical Signal Processing and Control, 41(2018) 69-80.

[24] Verma, R. and R. Pandey, Adaptive selection of search region for NLM based image denoising. Optik, 147(2017) 151-162.

[25] Elad, M. and M. Aharon, Image Denoising Via Sparse and Redundant Representations Over Learned Dictionaries. IEEE Transactions on Image processing.15(12)(2006) 3736-3745.

[26] Dabov, K., et al., Image Denoising by Sparse 3-D Transform-Domain Collaborative Filtering. IEEE Transactions on image processing, 16(8)(2007) 2080-2095.

[27] C. Tomasi, R. Manduchi, Bilateral filtering for gray and color images, in: Sixth Int. Conf. Comput. Vis. (IEEE Cat. No.98CH36271), Narosa Publishing House, (1998) 839-846. doi:10.1109/ICCV.1998.710815.

[28] A. Maykol Pinto, P.G. Costa, M. V. Correia, A. Paulo Moreira, Enhancing dynamic videos for surveillance and robotic applications: The robust bilateral and temporal filter, Signal Process. Image Commun. 29 (2014) 80-95. doi:10.1016/j.image.2013.11.003.

[29] D. Shao, P. Liu, D.C. Liu, Characteristic matching-based adaptive fast bilateral filter for ultrasound speckle reduction, Pattern Recognit. Lett. 34 (2013) 463-469. doi:10.1016/j.patrec.2012.12.006.

[30] W. Sun, A new single-image fog removal algorithm based on physical model, Opt. Int. J. Light Electron Opt. 124 (2013) 4770-4775. doi:10.1016/j.ijleo.2013.01.097.

[31] A. Phophalia, S.K. Mitra, Rough set based bilateral filter design for denoising brain MR images, Appl. Soft Comput. 33 (2015) 1-14. doi:10.1016/j.asoc.2015.04.005.

[32] Sadreazami, H., M.O. Ahmad and M.N.S. Swamy, A study on image denoising in contourlet domain using the alpha-stable family of distributions. Signal Processing, 128(2016) $459-473$.

[33] Sadreazami, H., M.O. Ahmad and M.N.S. Swamy, Color Image Denoising using 
Multivariate Cauchy PDF in the Contourlet Domain. 2016 IEEE Canadian Conference on Electrical and Computer Engineering, (2016) 1-4.

[34] Sadreazami, H., M.O. Ahmad and M.N.S. Swamy, Image denoising utilizing the scale-dependency in the contourlet domain.IEEE International Symposium on Circuits \& Systems. (2015) 2149-2152. DOI:10.1109/ISCAS.2015.7169105

[35] G. Noel, K. Djouani, B. Van Wyk, Y. Hamam, Bilateral mesh filtering, Pattern Recognit. Lett. 33 (2012) 1101-1107. doi:10.1016/j.patrec.2012.02.008.

[36] Visual Geometry Group, Datasets, Dep. Eng. Sci. Univ. Oxford. (2004). doi:http://www.robots.ox.ac.uk/ vgg/data3.html.

[37] Sadreazami, H., A.Asif and A.Mohammadi, Data-adaptive color image denoising and enhancement using graph-based filtering. IEEE Int. Symposium on Circuits and Systems (ISCAS), (9)(2017)1-4. 